\title{
Understanding veterinary leadership in practice
}

\author{
Caroline Elizabeth Pearson, Allan J Butler, Yaqub Paul Murray
}

The Vet Futures Report has identified 'exceptional leadership' as a key ambition for the long-term sustainability of the industry. This research investigates what it is like to be a veterinary surgeon in an in-practice leadership position, applying the qualitative methodology of interpretative phenomenological analysis. Through the researchers' interpretation of the seven participants' stories of their leadership experiences, the study advances understanding of the work environment, underlying motivations and the perceived responsibilities of veterinary leaders. Findings suggest, for many, a struggle in transition to leader positions, improving with time. The increase in pace of work is relayed by participants, with an ongoing, and unchallenged, work-life imbalance. The vets involved are highly motivated, driven by enjoyment of their jobs, a desire for self-determination and a need to make a difference. Relationships form the core of the perceived responsibilities, and yet are identified as the greatest day-to-day challenge of leadership. This study offers a valuable insight for veterinary surgeons, suggesting the industry could benefit from pausing and reflecting on behaviours. With a greater understanding of the complexity of leadership and followership, progress can be made to enact positive changes for the future.

\section{Introduction}

From early in employment, veterinary surgeons adopt a position of leadership, taking responsibility for case management, client communication and the coordination of the team of veterinary nurses and receptionists who facilitate their role. With time, many progress to become partners or directors within practice. A key ambition of the Vet Futures Report is 'exceptional leadership,' yet to date there has been little formal leadership training within the UK veterinary curriculums and a notable absence of veterinary-specific leadership literature. ${ }^{1}$ This vacuum in identifying the qualities of exceptional leadership raises the question of how the industry continues to progress, embracing the challenges of corporate ownership, growing numbers of veterinary surgeons and a turbulent macroenvironment during, and post, Brexit negotiations. ${ }^{2}$

Historically, leadership research has tended to follow a managerialist path, ${ }^{34}$ focusing analysis on alpha white men within US leadership..$^{5-7}$ As such, ethnocentric leadership models pervade the literature. Critically,

Veterinary Record (2018)

Department of Business Management and Entrepreneurship, Royal Agricultural University, Cirencester, UK

E-mail for correspondence: caroline@progressivevetconsulting. com doi: $10.1136 /$ vr. 104485

Provenance and peer review Not commissioned; externally peer reviewed.

Received April 28, 2017

Revised December 7, 2017

Accepted January 19, 2018 these models ignore contextual variation, the influence of leadership on the individuals within an industry and complexity. ${ }^{6}$ Therefore, in recent decades, literature has redirected to take a critical approach. In beginning to understand the value of leadership studies to the veterinary industry, the interconnection of leaders, followers, context and purpose in each unique leadership situation needs to be fully appreciated. ${ }^{8}$ Furthermore, to ensure exceptional leadership, we must first recognise its inseparability from the wellbeing of veterinary surgeons.

Given the Vet Futures' ambition of 'exceptional leadership' coupled with the paucity of literature on leadership in the veterinary industry, this study explores the experiences of leadership among senior veterinary surgeons (SVS), while acknowledging the complexity of multifactorial influence and uniqueness of practice. The value lies in reflecting on industry relevance. ${ }^{9}$

\section{Materials and methods}

Individuals have a unique interpretation of their experience within a specific context. ${ }^{10}$ Therefore, to understand leadership within the veterinary industry, this study uses qualitative research to recognise the subjective nature of knowledge and experience. A qualitative analysis can examine complex phenomena, such as leadership, and has received increasing recognition within healthcare and veterinary research. ${ }^{11} 12$

\section{Methodology}

Specifically, this research applied an interpretative phenomenological analysis (IPA) as it allows SVSs to 
make sense of their own experiences without preconception of causal theory. ${ }^{9}$ IPA is a detailed analysis of a small number of participants' stories. ${ }^{9}$ Individual case study helps to answer how or why questions ${ }^{13}$ and is particularly appropriate for understanding under-researched topic areas. ${ }^{14}$ The application in this paper is to address three research questions:

1. How do SVSs describe and manage the impact of work?

2. What motivations inform the way in which SVSs enact their leadership choices?

3. What are the perceptions held by SVSs about their leadership responsibilities?

Limitations

IPA has the dual purpose of relaying the personal experience of the participant (phenomenology) and questioning the data to enrich understanding (hermeneutics). Therefore, the researcher is heavily invested in the study, and the potential for personal bias and preconception during data collection and analysis is acknowledged. ${ }^{9}{ }^{15}$ However, full transparency of the practical aspects of the research (as detailed throughout) addresses validation such as authenticity and credibility. ${ }^{3}$

While generalisability in qualitative research is not possible, IPA provides a rich extension of the objective knowledge already in our possession, allowing relatability and transferability. ${ }^{49}$

\section{Methods \\ Sampling}

A small, purposive, convenience sample of seven SVSs was selected. Participants represented both genders and included farm animal, small animal and equine clinicians from independent veterinary practices. All had the common variable of a minimum of five years' experience as a veterinary leader, qualifying them to relate their unique experience of in-practice leadership. The researcher (herself a veterinary surgeon) knew all SVSs professionally or socially. Therefore, to address bias and preconception, only interview data were included in the analysis, excluding any prior knowledge of participants from interpretation (known as bracketing).

\section{Data collection}

Data collection involved semistructured interviews, using an interview template of open questions to prompt discussion of career history, current work behaviour, motivations, positives and negatives of role, and leader responsibilities. ${ }^{16}$ To mitigate effects of interview context on data, all participants chose a location where they felt most at ease to speak honestly, at a time when they had no additional work or social obligation.

Interviews were double-recorded to focus full attention on the SVSs' responses. The participants were urged to relate and reflect on what they felt was important to their lived experience of veterinary work and leadership. On completion of the interview, data were transferred verbatim to a line-numbered transcript and reviewed by the participant for accuracy, supporting construct validity ${ }^{17}$ Further contact with two participants on the intended meaning of one phrase within their transcripts enabled improved clarity.

\section{Data analysis}

Interviews were analysed sequentially, bracketing previous findings, to reduce the possibility of one interpretation influencing the next. A detailed, line-by-line, coding of each transcript was conducted, using descriptive, linguistic and conceptual annotation. ${ }^{9}$ Descriptive notes described what was evident from the data at face value, the content. Language use, tone, fluidity of speech and the use of metaphors formed the basis for linguistic description. The third level of coding allowed a deeper, interpretative focus, consistent with IPA. Questioning, self-reflecting and delving into underlying psychology of participants allowed an unveiling of more pertinent meaning. Throughout, it was important to maintain the link between interpretation and the transcribed words, avoiding imposition of preconceptions. ${ }^{9}$

Interpretation and data reduction continued to the identification of emergent discourses, capturing the importance of each participant's experience. Once completed for all SVSs, cross-case analysis highlighted convergence and divergence. ${ }^{14}$ Finally, discourses common to all transcripts were related in the words of the researcher, providing an interpretation of the meanings SVSs apply to their experiences.

A case study database was created containing the interview template, transcribed interviews, initial analytical constructs and discourse groupings, allowing two independent academic assessors to access and evaluate the research for transparency and credibility of interpretation. ${ }^{13}$ Inter-rater reliability for discourse identification was high.

\section{Results}

Box 1 shows a summary of the data, identifying core emergent discourses or themes. Within the original research, each discourse is discussed and evidenced by multiple extracts from the seven transcripts, in relation to developing understanding on SVS leadership and reflecting on industry relevance. However, it should be noted that due to editorial constraints, only limited quotations are possible here to provide invaluable insight to the analysis of SVSs' experiences.

SVSs, senior veterinary surgeons.

\section{How SVSs describe and manage the impact of work Discourse of transition}

All participants talked extensively of leadership transition. There was marked divergence within the data, with three considering the transition a natural progression, "not doing anything differently" from their time as an assistant veterinary surgeon. Others relayed the 


\section{Box 1: Emergent discourses in veterinary leadership}

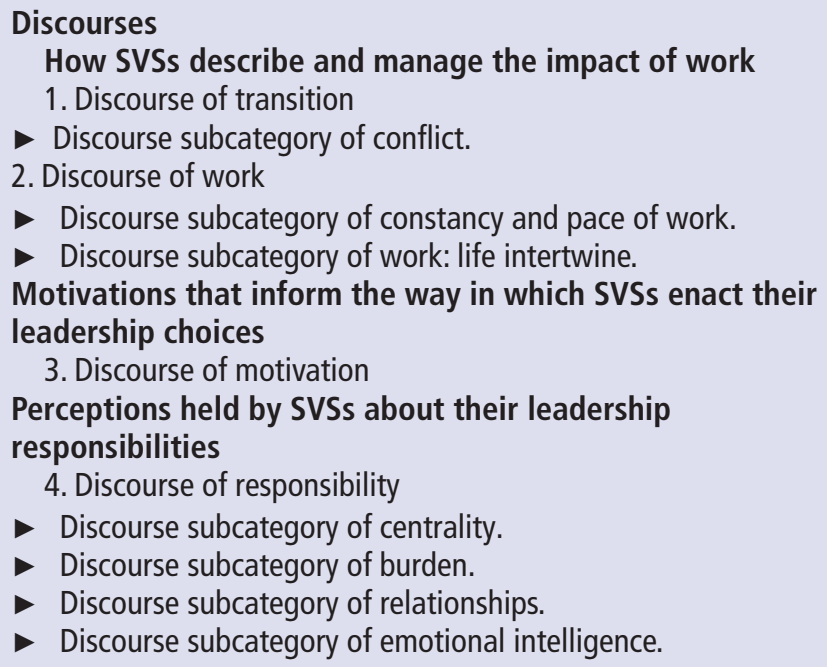

experience as stressful and traumatic, for example: "Well, you know, your role just goes full circle within 24 hours really. [ ], nothing really prepares you for it, [ ], it's making the transition overnight is, is very difficult. [], traumatic." (Note: [ ] indicates transcript wording omitted as they were not relevant to interpretation.)

There was an emphasis on the suddenness and lack of preparation for the transition to leadership. Each SVS related a time improvement in stress as they gained experience in their new position. However, several discussed conflicts among the leadership team as a major contributory factor to their experience: "I found the (intake of breath) partnerships meetings, the partnership meetings were very difficult, the confrontational nature. I've never known a group of people like that who were so combative."

The effects of conflict were increased stress, frustration and a feeling of low self-worth. Again, with time, the SVSs gained understanding of different personality types within the business and began to separate the conflict from personal assault, enabling a coping mechanism. However, at no point within the discourses was the conflict challenged as unacceptable, for example: "the failing is in the debate around the directors table [ ]. No point in trying to change things, but again, that is something that I am fine with now." For one participant, conflict was actively encouraged as a positive trait: "I've always encouraged all our directors to be open and honest [ ], almost to the point of being brutal. [] Works for me."

\section{Discourse of work}

The seven SVSs disclosed their ever-increasing workload and the pressure to keep pace, for example: "it's a constant juggling exercise." They accepted the need to maintain pace as part of current work culture and something they have no control over. While they acknowledged the potential negative effects of increasing

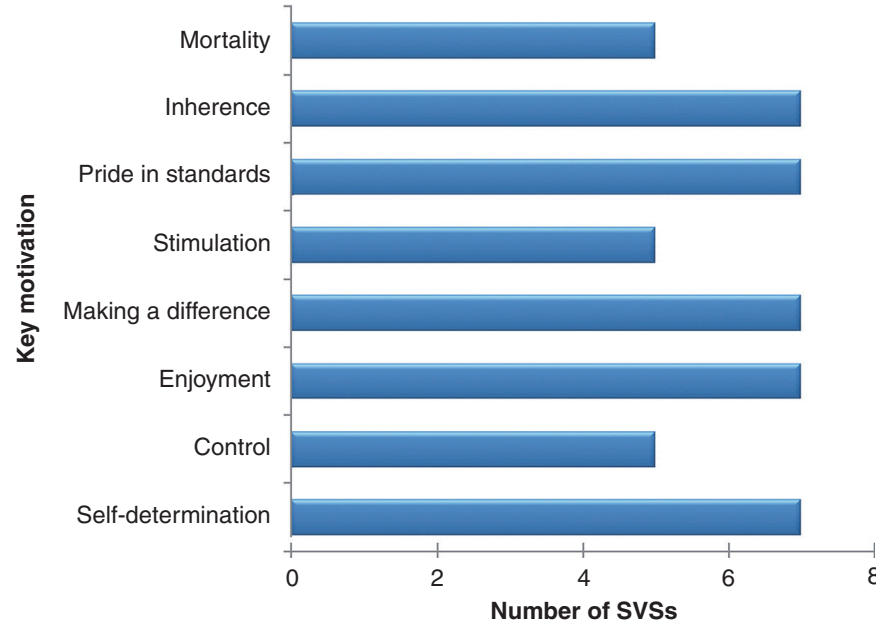

Figure 1: Senior veterinary surgeons' (SVSs) motivations for work behaviour.

workload, there was no evidence of efforts to change behaviour.

Each SVS discussed their struggle with work: life balance, but for some there was no separation, for example: "I live and dream my job so it is very much a way of life that can become all encompassing." Several participants described disturbed sleep patterns and negative effects on family life: "The work: life balance is obviously a difficult one...I perhaps could have done a... better job to emmm, rearing family...”

They justified negatives by citing benefits of increased remuneration and stability for family. Additionally, all relayed working long hours as an essential part of maintaining control and, therefore, reducing stress, for example: "you have to do it occasionally if you want to keep...So that you are not just constantly..., running flat out to stand still. That is a horrible way to be."

The transcripts elucidated an attachment to the SVS position beyond that of simply a job. In concurrence with research conducted by Page-Jones and Abbey, ${ }^{2}$ career appeared to be synonymous with identity, for example: "that's just who I am."

\section{Motivations that inform the way in which SVSs enact their leadership choices}

\section{Discourse of motivation}

Transcripts demonstrated considerable convergence with regard to motivations for leadership choices (figure 1).

Each participant cited enjoyment of their jobs as a primary motivator, for example: "A good day is when I am out on a farm, wearing wellies and talking to farmers, [ ] still beats everything hands down.” They also took great pride in standards, with a resultant frustration with those who did not pursue similar standards, for example: "It makes me really angry." Inherence is proposed as an underlying motivator, justifying work behaviours as beyond choice: "I think that is my character, being driven."

Five had a desire for ongoing stimulation, never reaching an endpoint of progression: "For me, that is 
what continues to make a job satisfying. It's developing and changing and progressing."

All seven expressed a need for self-determination and a desire to oversee their own destinies, without dependence on others, for example: "the more self-determination you have and the more you invest in it, I think, the more you are going to enjoy your work and your day to day life." For five, self-determination moved into the realm of control of others. Reflection allowed two participants to identify control as an attempt to reduce dissonance and stress, counteracting a struggle with delegation: "yeh, just an inability to delegate naturally and to think that you're, perhaps because you're the best person to do it, you're the only person to do it." However, for one SVS, there were repeated references to control of other's actions, and an attempt to shape staff and clients in their own likeness, for example: "You have to hope that you have built a team in your own mould."

Making a difference was discussed at length by all, providing a hope for existence and a purpose for continuance, for example: "it's just feeling like you make a positive contribution to the running of the business and I am not just there to exist."

Five discussed mortality as a motivator, reflecting on being defined in death by who they were in life, namely their veterinary work. For one SVS, excessive work was a means of delaying death, for example: "The day that I don't want to get out of bed in the morning will be the day that I give up. It will also be the day that I start towards my early grave. [ ] Enjoying the way that I work is potentially therapeutic as well as potentially harmful."

Finally, one SVS discussed a turnaround in motivation. An incidence of family ill health allowed a reassessment of priority away from work, back to home life. Acknowledgement of the temporality of life enabled a change of perspective and a reduction in stress: "why worry about stuff that is not important when you don't know what is going to happen tomorrow which would be way, way, worse than what's happened yesterday."

\section{Perceptions held by SVSs about their leadership responsi- bilities}

Discourse of responsibility

Centrality and burden

All participants identified high levels of responsibility. However, four emphasised they were central to the success of the business. Three claimed supremacy in decision making and team strengths, for example: "I think in the years that I was a partner we went from that to one of the top [ ] practices in the country and a lot of that came from, I feel, my drive and my ideas," or "I think I have made a difference. [ ] The teams I have built around me are really great teams and better than anyone else's teams." While strong commitment to staff and clients was noted, unquestioned self-aggrandisement and a failure to appreciate the contribution of others were evident.
For one SVS, centrality was related as a negative, resulting in a burden of responsibility and an isolation from support. He cited the situation as unsustainable, martyring himself for the industry, for example: "You can feel pretty rung out, pretty tense, eh, tired, quite hopeless and eh, I think you feel pressured and it can be quite lonely. I think there are only so many hours in the day and so many things an individual can do before they break."

\section{Relationships}

All transcripts acknowledged a leadership responsibility to maintain positive relationships with staff, colleagues and clients. Two-way respect was perceived vital and something to be earned. Diversity was discussed as undervalued within the industry, preferring group fit, for example: "we have been very good at going "no that is the shape that it is and if you want to be that shape you go to the practice with that shape, we are this shape'. [ ] the potential that that might bring has not really been appreciated.”

There was discussion with regard to the interaction between the whole and the parts of the business. All describe their responsibility to make choices for "the greater good" of the business. One provides a story of growing as a team together, appreciating the importance of every role for business and client success and personal satisfaction. The value of time invested in dialogue, training and respect was far beyond that of monetary gain. For example: "I would want them to understand why they were doing it so...ehhhhhh I would...I spent some time teaching them how to []. Keeping their enthusiasm and their reward. Much better than a pay rise [ ]. Because they were learning all the time, I think your jargon would be, they are on the same journey as you and the whole thing is just...going forwards..."

Empowerment and engagement flowed, yet this SVS claimed to have left the role of leadership to his business partners: "There has always been someone who wanted to be the king. I was happy to let them get on with it." He confused management and leadership. ${ }^{5}$ Nonetheless, the authentic leadership evident within his transcript was undeniable.

Others diverged to discuss the frustration and struggle of understanding and relating to people, for example: "that's one of the hardest things." A lack of mentoring was described as "a gaping hole in the lives of veterinary surgeons." Leadership was referred to as "intangible" and "something that has passed us by completely." Inadequate time to invest is cited as one reason for failed relationships, yet, in a profession full of committed and academically gifted people, transcripts alluded to a further deficiency.

\section{Emotional intelligence}

Several SVSs directly discussed emotional intelligence (EI) within the interview, for example: "There are some extremely skilled people in charge of the practice, some 
of them are not particularly emotionally astute, some are completely lacking in empathy, some are highly autocratic, and some have the view that it is my way, or you can just lump it." Hierarchical dominance, lack of empathy and inflexibility were identified as inadequacies within the leadership team. It was suggested the failure of understanding of others was a primary reason for high staff turnover, "the revolving door of staff." Additionally, contradictions within transcripts pointed to a struggle with self-understanding, some refusing to engage in personal reflection, for example: "Leadership is dealing with what is in front of you rather than feeling anything."

All SVSs described a time improvement in their EI capabilities due to parenthood and life experience, for example: "You can't just go on at 100 miles per hour all the way. You have to start to rely on your experience and take a more, steady approach.”

\section{Discussion}

Due to small sample size, the purpose of IPA research is not to generalise industry-wide. Instead, value lies in the transferability and relatability of narrative, as the reader reflects on and compares their experience with that of the participants. ${ }^{9}$ As such, a return to wider literature gives a more complete understanding of the identified convergence and divergence of stories. Three themes are particularly appropriate for this: impact of work, motivations and responsibility.

\section{Impact of work}

The divergent experience of transition to leadership is akin to the work of Kübler-Ross ${ }^{18}$ on change. While normal to experience a range of emotions because of change, the length of time spent in each phase varies for individuals due to many factors, such as context, emotions, politics, psychodynamics and mindsets ${ }^{19}$ (figure 2).
In this research, individual responses to adjustment to the leader position also varied. Notably, the transition was smoother for those three SVSs who considered themselves responsible for a leadership contribution from the first day of employment. Their mindset was better prepared. Conversely, the effect of conflict within the leadership team was to prolong the adjustment period.

Shifts in leadership thinking encourage us to question what is good and bad within a leadership context. ${ }^{2021}$ It is surely contentious to accept conflict as standard and unchangeable. Exceptional veterinary leadership and the wellbeing of veterinary surgeons demand improved preparation and support.

Continuing the discussion of work impact, research has identified a damaging work-life balance as a common cause of stress among UK veterinary surgeons and nurses. ${ }^{22}$ Cultural conformity can dampen our ability to question this imbalance, as vets attempt to fit into the work group, establish career recognition or seek business success, as evidenced in the interview data. ${ }^{23} 24$ However, the current way of working is proposed to be unsustainable, resulting in burn-out. ${ }^{25}{ }^{26}$ As identified by the Vet Futures Report, if the veterinary industry continues along the path of unquestioned workload, there is the potential for a reduction in wellbeing and resultant negative effects on leadership. Young vets' disillusionment due to lack of support from bosses is further indication that a problem already exists. ${ }^{27}$ If exceptional leadership is to be achieved, improving understanding of wellbeing must continue.

\section{Motivations}

The commitment of the SVSs to their roles, their enthusiasm, enjoyment, pride and fulfilment support research on the strength of veterinary career identity. ${ }^{2}$ There are extracts evidencing authentic leadership, where the value and power of the entire team are acknowledged. ${ }^{28}$

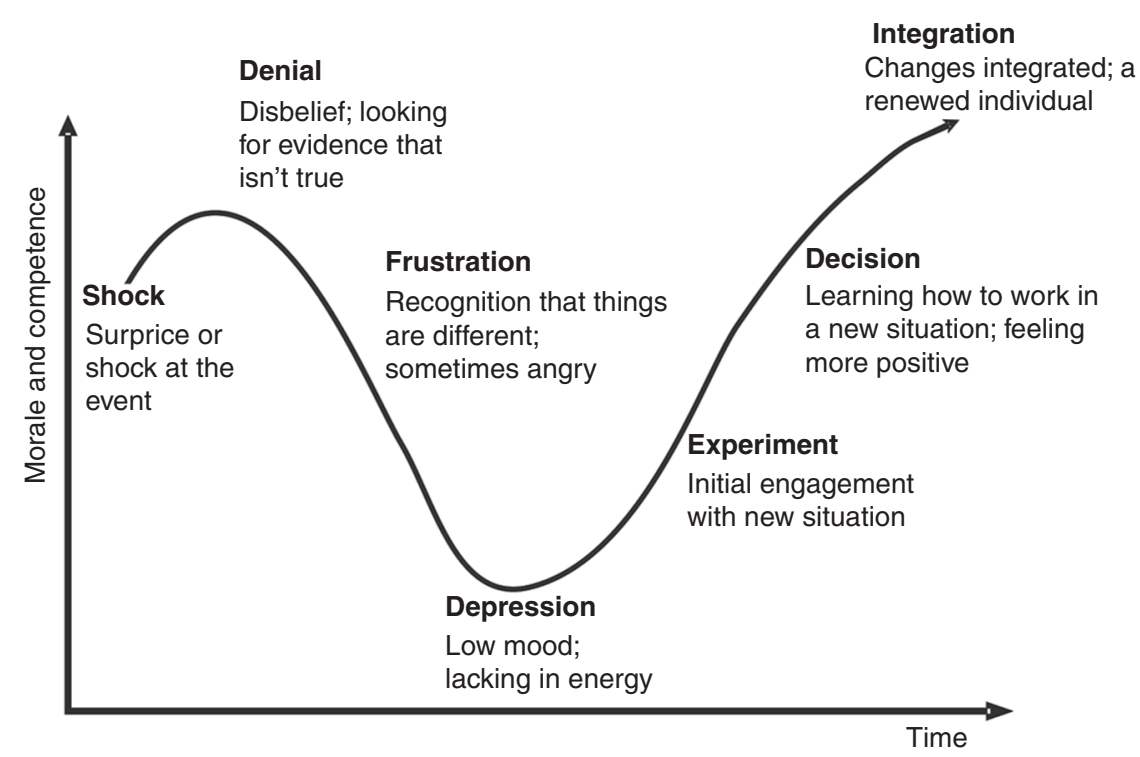

Figure 2: The 'Change Curve' diagram is adapted from the Elisabeth Kübler-Ross' 'Five Stages of Death' from On Death and Dying. Copyright 1969 by The Elisabeth Kübler-Ross Family LP. Reprinted by arrangement with The Elisabeth Kübler-Ross Family LP and The Barbara Hogenson Agency. All rights reserved. 
Based on interdependency, dialogic communication and ethical leadership, high personal reward and selfworth are the results, allowing the emergence of a progressive whole. ${ }^{29}$

The desire for self-determination is common within psychology literature as we attempt to reduce personal dissonance by maintaining control of our own futures. ${ }^{19} 30$ However, self-determination can move into the realm of control of others, as evidenced here, with the motivation of maintaining business profitability or overcoming delegation fear. ${ }^{31}$ The concern is, at best, the loss of potential of staff contribution. At worst, overcontrol opens the door to destructive leadership traits, such as narcissism. ${ }^{32}$ Narcissists demonstrate overconfidence, dominance, aggression, are unable to accept constructive dissent and have long-lasting effects on colleagues' wellbeing. ${ }^{33-36}$ Worryingly, narcissistic behavioural traits are more likely to be displayed during periods of stress or high workload. ${ }^{32} 333738$ If exceptional leadership is the aim, the challenge will be to recognise and prevent the symptoms of destructive leadership. ${ }^{32}$

Kets de Vries ${ }^{25}$ work on death (or existential) anxiety provides further understanding to the motivation of making a difference. By engaging in manic activity with 'purpose', we convince ourselves that our life has meaning. The aim is to produce something to outlive us and, so, extend our existence. However, when this behaviour becomes excessive, no degree of activity is enough. Within the transcripts, there is evidence of existential anxiety as SVSs describe work as ever-increasing, therapeutic and warding off death. Van Deurzen ${ }^{39}$ argues we find the courage to live fully if we come to terms with its temporality. Authentic leaders, who have faced the reality of death, embrace life, accepting responsibility and equality. ${ }^{10}$ The SVS who has regained perspective after family illness is a living example of the theory. While the participants may not fully comprehend the reasons for their behaviour, the parallels are significant. Therefore, for exceptional leadership to progress, perspective must be gained through self-reflection, accountability, inclusion and shared responsibility. Clarke et al ${ }^{12}$ support the need for a different veterinary mindset, citing benefits of improved social skills and an acceptance of complexity and uncertainty, facilitating a more rewarding career.

\section{Responsibilities}

All participants demonstrated high commitment. However, the theme of personal centrality to the overall success of the business highlights, for some, an inadequate understanding, or a narcissistic suppression, of the beneficial contribution of staff. ${ }^{40} 41$ The potential result is increased stress and workload for the leader and a reduced morale and loyalty for the follower. ${ }^{40}$ Ladkin and Taylor ${ }^{29}$ suggest a necessity to encourage staff responsibility and engagement if organisations are to progress effectively. Leadership is more than a leader.
Follower, context and purpose all have a role to play. One SVS exemplified effective leadership by embracing the non-zero-sum benefits of the whole as greater than the individual parts. ${ }^{42} 43$

Alongside the SVSs' acknowledgement of the importance of relationships built on respect, the struggle to understand themselves and others presents an ongoing challenge. The benefits of diversity within their organisations are unrealised. Restricted by time pressure and inadequate EI, there is evidence of unintentional destructive leadership behaviours, for example, inflexibility, avoidance, lack of empathy, callousness, insularity and unaccountability. ${ }^{3644}$ While literature continues to debate the definition, assessment and application of EI within organisations, the identification of the issue warrants further veterinary investigation, acknowledging relationships as an essential part of successful and ethical leadership. ${ }^{45-47}$

\section{Conclusion}

Historically, low priority has been given to veterinary leadership, resulting in a confused and inadequate understanding of the phenomenon's essential nature. The Vet Futures Report identified exceptional leadership and the wellbeing of veterinary surgeons as central to the long-term sustainability of the industry. ${ }^{1}$ However, emphasis is placed on the need for visionary, transformational industry leaders rather than the day-to-day challenge of leadership within practice.

This paper offers valuable insight into the challenges of attaining the ambition of exceptional leadership. For improvement, we must first comprehend what it is like to be an SVS, challenging the effects of increasing workloads and destructive behaviour. Reflection on personal motivation, improved understanding of others and focus on the means, rather than the end, will progress inclusive and ethical leadership, embracing the benefits of empowered, diverse and valued teams. ${ }^{48} 49$ Further research into the effects of SVS leadership choices is warranted, alongside investigations of responsible followership, promoting the holistic aim of exceptional leadership and wellbeing for all.

Acknowledgements The authors would like to thank the veterinary surgeons for giving their time and stories, providing a vital insight into the challenges of veterinary leadership.

\section{Competing interests None declared.}

Ethics approval Ethical approval was obtained from the ethics review committee at the institution of research.

(C) British Veterinary Association (unless otherwise stated in the text of the article) 2018. All rights reserved. No commercial use is permitted unless otherwise expressly granted.

\section{References}

1 Vet Futures Board. Taking charge of our future: A vision for the veterinary profession for 2030. 2015 http://www.vetfutures.org.uk/resource/vet-futures-report/ (accessed 20 Oct 2015).

2 PAGE-JONES S, ABBEY G. Career identity in the veterinary profession. Vet Rec 2015;176:433

3 STENTZ JE, PLANO CLARK VL, MATKIN GS. Applying mixed methods to leadership research: a review of current practices. Leadersh Q 2012;23:1173-83. 
4 PARRY K, MUMFORD MD, BOWER I, et al. Qualitative and historiometric methods in leadership research: a review of the first 25 years of the leadership quarterly. Leadersh Q 2014; 25:132-51.

5 GRINT K. The cuckoo clock syndrome: addicted to command, allergic to leadership. European Management Journal 2010;28:306-13.

6 GREY C. A very short, fairly interesting and reasonably cheap book about studying organizations. 3rd edn: SAGE Publications Ltd, 2013.

7 CUNLIFFE AL. A very short, fairly interesting and reasonably cheap book about management. 2nd edn: SAGE Publications Ltd, 2014

8 LADKIN D. Rethinking leadership: a new look at old leadership questions: Edward Elgar Publishing Limited, 2010.

9 SMITH JA, FLOWERS P, LARKIN M. Interpretive phenomenological analysis: theory, method and research: SAGE Publications Ltd, 2009.

10 SPINELLI E. The value of relatedness in existential psychotherapy and phenomenological enquiry. Indo-Pacific Journal of Phenomenology 2006;6:1-8.

11 AL-BUSAIDI ZQ. Qualitative research and its uses in health care. Sultan Qaboos Uni Med / 2008;8:11-19.

12 CLARKE C, KNIGHTS D, FINCH G. Addressing disillusionment among young vets. Vet Rec 2016;179:603.1-4

13 DE MASSIS A, KOTLAR J. The case study method in family business research: guidelines for qualitative scholarship. Journal of Family Business Strategy 2014:5:15-29.

14 EISENHARDT KM. Building theories from case study research. Academy of Managemen Review 1989;14:532-50

15 MCQUARRIE EF, MCINTYRE SH. What can you project from small sample qualitative research? Marketing Insights 2014:26:34-9.

16 JACOB SA, FURGERSON SP. Writing interview protocols and conducting interviews: tip for students new to the field of qualitative research. Qualitative Report 2012;17:10.

17 YIN RK. Case study research: design and methods. 5th edn: SAGE Publications, Inc 2014.

18 KÜBLER-ROSS E. On death and dying. The Macmillan Company, 1969.

19 KETS DE VRIES MFR, MILLER D. The neurotic organization. Jossey-Bass Inc, 1984.

20 KELLERMAN B. Bad leadership: what it is, how it happens, why it matters. Harvard Business School Press, 2004.

21 ERICKSON A, SHAW B, MURRAY J, et al. Destructive leadership. causes, consequences and countermeasures. Organizational Dynamics 2015;44:266-72.

22 BUZZEO J, ROBINSON D, WILLIAMS M. The 2014 RCVS survey of the veterinary profes sion. Institute for Employment Studies, 2014

23 TOURISH D, PINNINGTON A. Transformational leadership, corporate cultism and the spirituality paradigm: an unholy trinity in the workplace? Human Relations 2002;55:147-72.

24 TOURISH D, VATCHA N. Charismatic leadership and corporate cultism at Enron: the elimination of dissent, the promotion of conformity and organisational collapse. Leadership 2005;1:455-80

25 KETS DE VRIES M. Death and the executive: encounters with the "stealth" motivator. Organ Dyn 2014:43:247-56.

26 FIELD V. 'Proactive approaches to stress in the veterinary workplace'. Veterinary Ireland Journal 2011;64:84
27 Vet Futures. Disillusionment among young vets. causes and potential solutions. Vet Rec 2016;179:375-6.

28 RAYNER ADM. Inclusionality and the role of place, space and dynamic boundaries in evolutionary processes. Philosphica 2004;73:51-70.

29 LADKIN D, TAYLOR SS. Enacting the 'true self': Towards a theory of embodied authentic leadership. Leadersh Q 2010;21:64-74.

30 VINCE R, MAZEN A. Violent innocence: a contradiction at the heart of leadership. Organization Studies 2014;35:189-207.

31 AVOLIO BJ, WEBER TJ, WEBER TJ. Leadership: current theories, research, and future directions. Annu Rev Psychol 2009;60:421-49.

32 KRASIKOVA D, GREEN S, LEBRETON J. Destructive leadership: a theoretical review, integration, and future research agenda. Journal of Management 2013;39:1308-38.

33 ROSENTHAL SA, PITTINSKY TL. Narcissistic leadership. Leadersh Q 2006;17:617-33.

34 BABIAK P, HARE RD. Snakes in suits: when psychopaths go to work. Harper, 2007.

35 HIGGS M. The good, the bad and the ugly: leadership and narcissism. Journal of Change Management 2009;9:165-78.

36 SCHYNS B, SCHILLING J. How bad are the effects of bad leaders? A meta-analysis of destructive leadership and its outcomes. Leadersh Q 2013;24:138-58.

37 LIPMAN-BLUMEN J. Toxic leadership: a conceptual framework. Encyclopedia of Executive Governance 2005a:1-12.

38 LIPMAN-BLUMEN J. The allure of toxic leaders: why we follow destructive bosses and corrupt politicians- and how we can survive them. Oxford University Press, 2005b.

39 VAN DEURZEN E. From psychotherapy to emotional well being. Análise Psicológica 2006;24:383-92

40 KELLERMAN B. Followership: how followers are creating change and changing leaders. Harvard Business Review Press, 2008

41 UHL-BIEN M, RIGGIO RE, LOWE KB, et al. Followership theory: a review and research agenda. Leadersh Q 2014;25:83-104.

42 HIDALGO CA. Planet hard drive. Sci Am 2015;313:72-5.

43 HIDALGO C. Why information grows: Penguin, 2016

44 SHOTTER J, TSOUKAS H. In search of phronesis: leadership and the art of judgment. Academy of Management Learning \& Education 2014:13:224-43.

45 GARDNER WL, SCHERMERHORN JR. Unleashing individual potential: performance gain through posiitve organisational behaviour and authentic leadership. Organisational Dynamics 2004:33:270-81.

46 ZEIDNER M, ROBERTS RD, MATTHEWS G. The science of emotional intelligence: current consensus and controversies. European Psychologist 2008;13:64-78.

47 WEBB KS. Why emotional intelligence should matter to management: a survey of the literature. SAM Advanced Management Journal 2009;74:32-41.

48 GRANT AM, PARKER SK. 7 Redesigning work design theories: the rise of relational and proactive perspectives. Acad Manag Ann 2009:3:317-75.

49 GRANT A. How to build a culture of originality. Harvard Business Review 2016;94:86-94.

D Check for updates 